Research article

\title{
ROS signaling as common element in low oxygen and heat stresses
}

\author{
Chiara Pucciariello*, Valeria Banti, Pierdomenico Perata \\ PlantLab, Institute of Life Sciences, Scuola Superiore Sant'Anna, Piazza Martiri della Libertà 33, 56127 Pisa, Italy
}

\section{A R T I C L E I N F O}

\section{Article history:}

Received 14 December 2011

Accepted 17 February 2012

Available online 3 March 2012

\section{Keywords:}

Anoxia

Heat

Hypoxia

ROS

\begin{abstract}
A B S T R A C T
The activation of the oxidative metabolism in plants under low oxygen conditions has prompted controversial views. The presence of a ROS component in the transcriptome in response to low oxygen has been observed and an overlap with heat stress has been proved. It has been also demonstrated that ROS are produced during both anoxia and heat, but the site of their production remain contentious. Membrane NADPH oxidase and mitochondrial electron transport flow have been indicated as possible ROS generation systems. Both anoxia and heat have been shown to induce the transcription of Heat Shock Factors (HSFs) and Heat Shock Proteins (HSPs), among which HSFA2 and some of its targets. HSFA2 over-expressing plant has been shown to be more tolerant to anoxia, while the knockout $h s f a 2$ lose the capability of wild type plants to cross-acclimate to anoxia through mild heat pre-treatment. The production of ROS seems to be an integral part of the anoxia and heat response, where HSFs likely play a central role in activating the HSP pathway. This mechanism is suggested to result in enhanced plant tolerance to both anoxia and heat.
\end{abstract}

(c) 2012 Elsevier Masson SAS. All rights reserved.

\section{Introduction}

Many years of studies on plants that have been subjected to different biotic and abiotic stress conditions have suggested Reactive Oxygen Species (ROS) as ubiquitous markers of both oxidative stress and of signaling events for the induction of adaptive stress responses [64]. This dual function is tightly regulated by a balance between ROS production and scavenging, which is coordinated by a large gene network that is activated under stress conditions [63]. This equilibrium allows plants to maintain cell homeostasis, and also to accumulate ROS in subcellular compartments where they act as signals [64]. Various enzymes directly involved in the generation of ROS, such as the plant homolog of the mammalian respiratory burst NADPH oxidase (RBOH), have been identified and demonstrated to generate and amplify ROS production for signaling $[62,97]$. Organelles with a highly oxidizing metabolic activity or whose metabolism relies on electron transport (i.e. chloroplasts, mitochondria and microbodies) have also been found to be sources of ROS in plant cells and contribute to the oxidative burst during abiotic stresses [63,96]. ROS metabolisms in these organelles are also a source of retrograde signals to the nucleus that play important roles in stress acclimation [96].

Of the abiotic stresses, low $\mathrm{O}_{2}$ has prompted a controversial hypothesis regarding the activation of the oxidative metabolism,

\footnotetext{
* Corresponding author.

E-mail addresses: c.pucciariello@sssup.it, chiara.pucciariello@sssup.it (C. Pucciariello).
}

since it is seemingly illogical that an increase in ROS production is due to a decrease in the essential substrate $\mathrm{O}_{2}$. However, the presence of a ROS component in the transcriptome in response to low $\mathrm{O}_{2}$ has been suggested by many microarray studies [8,11-13,43,59]. Moreover, an overlap between the molecular response to $\mathrm{O}_{2}$ depletion and heat stress has been observed $[7,8]$. Thus it has been shown that, not only are the transcriptomic patterns similar between these two conditions, but Arabidopsis plants pre-treated with mild heat stress also have an increased tolerance to anoxia stress [7]. Indeed, transgenic Arabidopsis plants over-expressing the heat shock transcription factor (TF) HSFA2 show an enhanced anoxia tolerance [8]. Moreover, knockout $h s f a 2$ plants lose the ability to cross-acclimate to anoxia before a mild heat stress pre-treatment [8]. The activation of redox-sensitive TFs, such as heat shock (HSFs), has been proposed as one of the possible ROS-sensing mechanisms in plants and animals [60].

In this review, we provide an update of the most recent findings that support the presence of ROS signaling under low $\mathrm{O}_{2}$, and suggest that these molecules are the common factors that determine the overlap between anoxia and heat response. We also discuss controversial hypothesis of producing, sensing and signaling the oxidative metabolism under these stress conditions.

\section{Low oxygen stress and adaptive responses in plants}

Aerobic organisms require molecular $\mathrm{O}_{2}$ in order to efficiently produce ATP. Oxygen is the final acceptor of $e^{-}$in the mitochondrial 
respiratory chain, which is the last step in respiration that leads to energy synthesis through ATP production [5]. Oxygen depletion has a rapid and severe consequence on cells, which are altered in terms of their metabolism, energy production and consumption as well as gene expression. However, aerobic organisms have evolved adaptive responses that can compensate for an energy crisis and in some cases confer tolerance [4]. In plants, transient flooding events, waterlogging and microbial activity in the soil frequently and rapidly lead to $\mathrm{O}_{2}$ deficiency [29]. With an $\mathrm{O}_{2}$ deficit, ATP formation is severely reduced and a tight regulation of energy production occurs through the shift of respiration from aerobic to anaerobic. This change relies primarily on glycolysis and fermentation to generate ATP and regenerate $\mathrm{NAD}^{+}$to sustain glycolysis. However, an energy crisis follows since fermentation of one molecule of hexose yields only $2 \mathrm{~mol}$ ATP per mol hexose compared to up to $38 \mathrm{~mol}$ ATP produced by aerobic respiration. Moreover, both a short initial lactic and a long-lasting ethanol fermentation occur after glycolysis [73]. The accumulation of lactate can impair plant survival under water, since its dissociation seems to contribute to the acidification of the cytosol [109]. Under hypoxia, cells are still capable of producing ATP via oxidative phosphorylation, although consuming less $\mathrm{O}_{2}$ and reorganizing metabolic fluxes for a more focused use of energy $[53,83]$. Together with a metabolic adjustment, plants adapt to $\mathrm{O}_{2}$ limiting conditions through morphological modification. In rice, variety-specific adaptations include either rapid internode-leaf elongation to escape submergence and restore the contact with the air [35], or induction of a quiescent state to reduce the growth to a minimum under unfavorable conditions [25]. The formation of aerenchyma to facilitate gas exchange $[19,72]$, the production of adventitious roots on stem areas [110] and the hyponastic growth of leaves [20] are only some of the morphological traits that contribute to the plant's ability to survive low $\mathrm{O}_{2}$. In addition, molecular reprogramming has been examined through several genome-wide analyses of many plant species exposed to different levels and extents of $\mathrm{O}_{2}$ reduction $[12,41,49,51,56,59,67,108]$. Some of the transcripts modulated under $\mathrm{O}_{2}$ depletion correspond to ROS detoxification enzymes, ROS-regulated TF and Heat Shock Proteins (HSPs), suggesting that ROS contribute to low $\mathrm{O}_{2}$ signaling.

\section{Secondary ROS signal in plants under low oxygen}

Plant sensing of $\mathrm{O}_{2}$ deprivation is a key aspect in adaptation. Recently, much progress has been made in understanding the mechanism behind low $\mathrm{O}_{2}$ sensing, and a direct sensing mechanism of $\mathrm{O}_{2}$ availability in Arabidopsis has been described $[30,54]$. The conserved N-terminal amino acid sequence Met-Cys present in some Ethylene Responsive Factors (ERF) of group VII (e.g. Arabidopsis HRE1, HRE2, RAP2.2 and RAP2.12) is the target of an ubiquitin-dependent protein degradation under normoxic conditions, through the oxidation of a Cys residue after Met cleavage [30,54]. Stabilization of the Met-Cys N-terminal motif under low $\mathrm{O}_{2}$ with the consequent TF migration to the nucleus [54] leads to enhanced plant survival, through the control of the expression of core hypoxic genes related to the anaerobic metabolism such as $A D H 1, P D C 1$, and SUS4 [30]. It is still unclear whether the N-terminal Met-Cys stability depends directly on $\mathrm{O}_{2}$ or cellular changes associated with its availability, such as cytosolic pH or ROS balance [30].

Interestingly, transcriptome adjustment under low $\mathrm{O}_{2}$ also includes other genes that are likely not directly regulated by the $\mathrm{N}$ end rule mediated oxygen sensing. A survey of microarray data produced under low $\mathrm{O}_{2}$ has shown that a conserved group of HSPs was expressed together with the group of core genes related mostly to anaerobic metabolism $[8,59,67]$. Unlike the core of anaerobic genes, these HSPs are not constitutively expressed in the N-end rule Arabidopsis mutants such as ate $1 / 2$ and prt6 that lack some of the recognition steps required for proteasomal degradation [30,31]. ate 1/2 lacks the arginyl-tRNA-protein transferase (ATE1 and ATE2) required to arginylate the oxidized Cys [120]. prt6 lacks the E3 ligases called N-recognins (PRT6) that recognize the primary destabilizing residues for proteasome degradation [27]. Rather than directly depending on direct low-oxygen sensing, the induction of these HSPs under low $\mathrm{O}_{2}$ seems instead to be dependent on an $\mathrm{H}_{2} \mathrm{O}_{2}$ burst that is observed early after the onset of $\mathrm{O}_{2}$ deprivation [8]. Thus, it is tempting to speculate that the up-regulation of ROS-related proteins such as HSPs, but also TFs such as ZAT10 and ZAT12, which are known to be modulated under low $\mathrm{O}_{2}$ $[8,56,57]$, constitute a specific response under the control of a secondary signaling mechanism that may rely on redox modifications (Fig. 1).

Recent results have demonstrated that the oxidative burst is a common theme in heat and anoxia stress [8]. Of the TFs transcriptionally regulated by both conditions, HSFA2 is the most responsive to $\mathrm{H}_{2} \mathrm{O}_{2}$ [60], and is one of the TFs that are commonly up-regulated in different experiments related to ROS production in different plant cell compartments [26]. In eukaryotes, HSFs have been suggested to be direct sensors of the ROS that modulate their activity [60]. Hydrogen peroxide has been found to promote a shift from the inactive monomers to the active homotrimer form of mammalian and Drosophila HSF1, likely via the redox regulation of the disulfide bound formation in the Cys residues located within the DNA-binding domain of the TF $[3,122]$. Superoxide anion is, on the other hand, responsible for the conformational change in Saccharomyces cerevisiae HSFs trimeric form to the activated state [50]. The S. cerevisiae response regulator $\mathrm{Skn} 7$ has been proposed to require $\mathrm{H}_{2} \mathrm{O}_{2}$ to interact with $\mathrm{HSF} 1$ and activate $H S P$ s transcription [76]. Two hypothetical models of HSFs action in plants have been proposed by Miller and Mittler [60]. In the first, $\mathrm{H}_{2} \mathrm{O}_{2}$ regulates the constitution of the HSFs homotrimer form which acts as a transcriptional activator, in the second, the homotrimeric form of a particular HSF interacts with a second $\mathrm{HSF}$ via $\mathrm{H}_{2} \mathrm{O}_{2}$ mediation to induce gene expression [60]. Indeed, plants' HSFs have been found to cooperate to regulate downstream events $[6,18,87]$, however whether $\mathrm{H}_{2} \mathrm{O}_{2}$ directly functions as activator in plants is still unclear [58].

The presence of an oxidative burst under anoxia has been reported by Baxter-Burrel et al. [9], who showed that the activation of Rop2, a RHO-like small G protein of plants (Rop), under low $\mathrm{O}_{2}$ contributes to $\mathrm{H}_{2} \mathrm{O}_{2}$ accumulation. This was shown to occur via a NADPH oxidase mechanism, which is also required for $A D H$ expression [9]. Dominant negative Rop2 ( $D N$-rop2) seedlings under $\mathrm{O}_{2}$ deprivation showed neither transcript accumulation nor activity of ADH but increased sensitivity to stress [9]. The activity of Rop2 is likely to be controlled by a negative feedback regulation which requires a $\mathrm{H}_{2} \mathrm{O}_{2}$-regulated Rop GTPase activating protein (GAP), RopGAP4, that preferentially stimulate the GTPase activity of Rop and thus inactivates Rop2 [9]. The disruption of the RopGAP4 in ropgap4-1 seedlings increases $A D H$ expression but decreases tolerance to $\mathrm{O}_{2}$ deprivation. Thus, tolerance to low $\mathrm{O}_{2}$ requires both Rop2 activation and RopGAP4-dependent negative feedback regulation which modulates $\mathrm{H}_{2} \mathrm{O}_{2}$ production and $\mathrm{ADH}$ activity and expression [4,9]. Whether this mechanism might in part overlap with the $\mathrm{N}$-end rule pathway in regulating $A D H$ expression is still unclear (Fig. 1). The physiological and biochemical nature of the signal that activates this pathway remains speculative.

In animals, the heterodimer TF Hypoxia Inducible Factor 1 (HIF1) has been identified as a direct sensor and regulator of transcriptional responses under hypoxia [38,39]. Mammalian HIF-1 is 
Oxygen level

ROS

signal
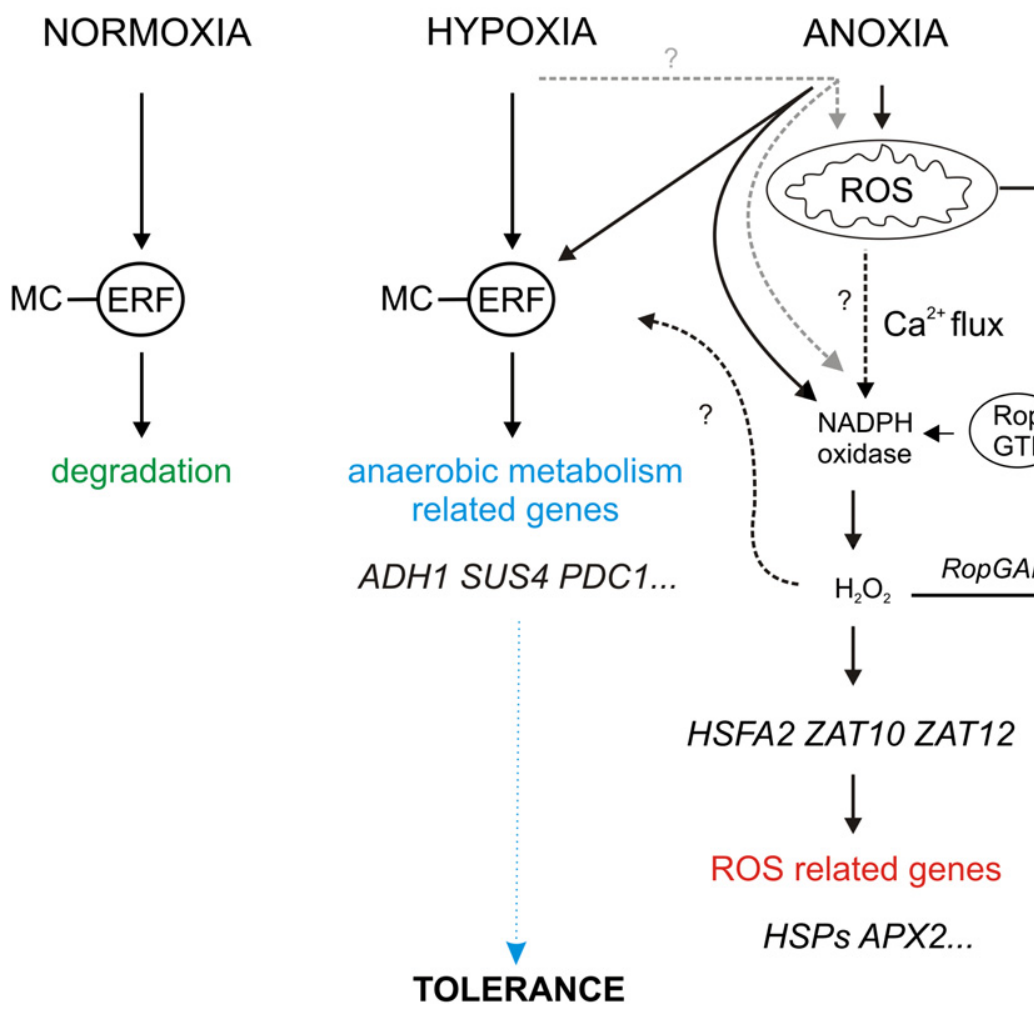

MAPK3/6 production

dogradation

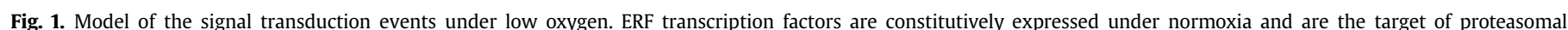

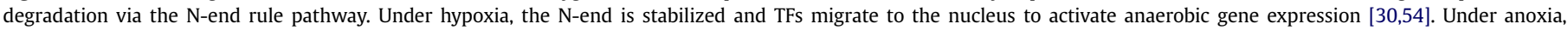

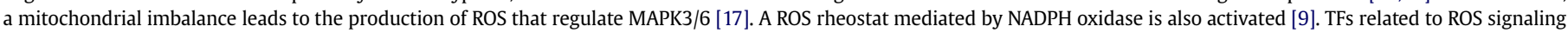

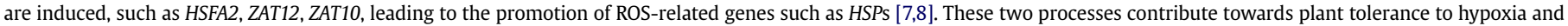
anoxia.

made up of the hypoxia-induced HIF-1 $\alpha$ subunit and the constitutively expressed HIF-1 $\beta$ one. Under aeration, HIF-1 is not active, since HIF- $1 \alpha$ is hydroxylated at two prolyl residues by the prolyl-hydroxilase (PHD) enzymes which require $\mathrm{O}_{2}$ as a cosubstrate. The aerobically hydroxylated HIF- $1 \alpha$ is degraded via the ubiquitin-mediated proteasome degradation through the recognition of hydroxylation tags by the von Hippel-Lindau (VHL) tumor suppressor $[37,69]$. Reduced $\mathrm{O}_{2}$ availability reduces the rate of HIF- $1 \alpha$ degradation with the subsequent activation of the transcriptional complex responsible for the activation of the transcription of a large number of hypoxia-related genes [91]. The exogenous or endogenous induction of ROS under normoxia is known to stabilize HIF-1 $\alpha[15,32]$. In addition, treating mammalian cells with antioxidants abolishes the HIF-1 response [14,32], thus suggesting a role for ROS in controlling HIF-1 action. In plants, no orthologs of the animal HIF have been found so far [55].

\section{Enigmatic site of ROS production under oxygen depletion}

\section{1. $R B O H-N A D P H$ oxidase function under low oxygen in plants}

Reactive oxygen species are by-products of normal cellular metabolism with high oxidizing activities such as photosynthesis, respiration and photorespiration. They are also directly produced by enzymes, which include oxalate and amine oxidase, peroxidases and NADPH oxidases (RBOHs). RBOHs likely play a key role under several stress conditions [97], regulating ROS signaling in response to heat, drought, cold, high-light, salinity and wounding, but also biotic stresses and developmental processes [97]. RBOHs produce $\mathrm{O}_{2}^{-}$by oxidizing $\mathrm{NADPH}$ and transferring electrons to $\mathrm{O}_{2}$ at the plasma membrane $[61,85,100]$. The oxidative burst which produces ROS via this system occurs in the apoplast [103]. Under low $\mathrm{O}_{2}$, plants treated with diphenylene iodonium chloride (DPI), which is an inhibitor of flavincontaining NADPH oxidases, show a reduction in ADH activity, thus indicating that ADH action requires a DPI-sensitive NADPH oxidase [9]. DPI treatment reduces the low $\mathrm{O}_{2}$ stress tolerance of wild type Arabidopsis plants, but also increases the survival of ropgap4-1 seedlings, thus showing that low $\mathrm{O}_{2}$ tolerance needs either the activation or deactivation of DPI-sensitive NADPH oxidase [9]. RBOHD is known to mediate many processes such as (a)biotic stress responses, stomatal movements, lignification $[23,47,61,101,102]$. It is transcriptionally regulated under some abiotic stresses [97], and its up-regulation is observed under low $\mathrm{O}_{2}$. rbohD and rbohB mutants have been shown to be sensitive to heat stress [48]. ROS generated by RBOHD could be a common response between heat and anoxia.

A relationship between $\mathrm{RBOH}$-dependent ROS production and $\mathrm{Ca}^{2+}$ homeostasis has been suggested in Arabidopsis [97]. It is likely that $\mathrm{Ca}^{2+}$ binds to the EF-hands of the N-terminal region of $\mathrm{RBOH}$ 
and, together with RBOH phosphorylation, promotes ROS production, with the subsequent activation of $\mathrm{Ca}^{2+}$ channels in a positive feedback amplification [68,97,100]. Transfer to anoxia has been shown to induce $\mathrm{Ca}^{2+}$ modulation in Arabidopsis, maize and rice. In maize, the induction of $A D H$ under anoxia is likely to require an increase in cytosolic $\mathrm{Ca}^{2+}[94,95]$. In Arabidopsis and rice, the oscillation of cytosolic $\mathrm{Ca}^{2+}$ was observed following transfer to anoxia, with $\mathrm{Ca}^{2+}$ channel blockers partially inhibiting the $A D H$ anaerobic induction [90]. Since a cytosolic increase in $\mathrm{Ca}^{2+}$ is a prerequisite for the activation of $\mathrm{H}_{2} \mathrm{O}_{2}$ production by $\mathrm{Ca}^{2+}$ dependent NADPH oxidase, it is tempting to speculate a connection between Arabidopsis Rop-promoted $\mathrm{H}_{2} \mathrm{O}_{2}$ production [9] and cytosolic $\mathrm{Ca}^{2+}$ increases [90] in regulating ADH expression and activities (Fig. 1).

\subsection{The role of mitochondria in ROS production under low oxygen:} evidence from animals and plants

Mitochondria represent the major sink of cellular $\mathrm{O}_{2}$. It is thus reasonable to suppose that this organelle is equipped with an $\mathrm{O}_{2}$ availability sensing mechanism [116]. The nature of the hypoxic signal produced by mitochondria is still under debate, since it may involve the cell energy state, the redox homeostasis, and also the direct production of ROS [32].

In mammalian cells belonging to vascular tissues, the role of mitochondria in ROS production under low $\mathrm{O}_{2}$ has been hypothesized [116,118]. Although related to very specific structures (e.g. pulmonary and systemic arteries cells in response to alveolar hypoxia) these hypothesis could furnish suggestions for other less specialized systems. One hypothesis is that severe hypoxia limits the mitochondrial $e^{-}$chain at complex IV, where $\mathrm{O}_{2}$ is reduced to $\mathrm{H}_{2} \mathrm{O}$. Oxygen deprivation at complex IV might affect the redox state of the upstream $e^{-}$carriers and initiate redox-signaling downstream events [118]. Under normoxia, mitochondria complexes produce $\mathrm{O}_{2}^{-}$, not only in the mitochondrial matrix but complex III also in the intermembrane space $[32,66,104]$. In this latter subcellular compartment, an over-production of ROS as consequence of homeostasis disruption may be more likely to reach the cytosol and act as a signal [32,33]. Superoxide can spontaneously, or via SOD, dismutate to yield $\mathrm{H}_{2} \mathrm{O}_{2}$, which as a non-charged molecule, can likely diffuse through the mitochondrial membranes $[33,104]$. Recently, the fluorescent redox sensor roGFP was used to provide a measure of the protein thiol redox state in the mitochondrial matrix, the intermembrane space, and the cytosol in mammalian cells [117]. During hypoxia, roGFP oxidation in the cytosol and the intermembrane space showed an oxidative shift, indicating ROS production in that cell compartment [117]. This hypoxiainduced ROS production was reduced by over-expressing cytosolic catalase, thus demonstrating $\mathrm{H}_{2} \mathrm{O}_{2}$ involvement in the process [117].

The use of specific inhibitors has further led to the suggestion that the critical step for ROS generation in mammalian cells under low $\mathrm{O}_{2}$ resides in mitochondria at Complex III [14]. Indeed, while Complex I inhibitors, such as rotenone, and $Q_{0}$ site of the Complex III inhibitors, like myxothiazol (MYX), were able to suppress the rise of HIF- $1 \alpha$ under low $\mathrm{O}_{2}$, the $Q_{\mathrm{i}}$ site inhibitor antimycin A (AA) had no effect on the subunit rise [14,15,32].

Very recently, evidence of mitochondria-associated ROS production involvement in response to $\mathrm{O}_{2}$ deprivation in Arabidopsis seedlings has been demonstrated [17]. Upon $\mathrm{O}_{2}$ deprivation and also reoxygenation, the rapid and transient activation of mitogen-activated protein kinases (MPK) 3, 4 and 6 has been found to occur and to be activated by mitochondrial ROS. Treatment of normoxic plants with mitochondrial ETC inhibitors acting at complex III (AA) and at complex IV (potassium cyanide, KCN) also showed MPK6 and 3 activation, together with ROS production in mitochondria [17]. Both AA and KCN promote ubisemiquinone accumulation, increasing ROS formation. Moreover, both AA and KCN pre-treatment decreased MPK6 and 3 activation when seedlings were transferred to $\mathrm{O}_{2}$ deprivation or reoxygenation, sustaining the link between mitochondrial ROS and MPK activation [17]. MPK cascades are known to be regulated by several stresses that have an oxidative component $[2,40,114,121]$. The overexpression of MPK6 leads to enhanced survival to $\mathrm{O}_{2}$ deprivation, but not significant modulation of classic transcripts related to the anaerobic metabolism, thus suggesting a benefit for plant survival that is probably related to another pathway [17].

Indeed, a link between functional mitochondria and HSP production has been shown [81]. Arabidopsis cell treatment with mitochondrial inhibitors and uncouplers during mild heat shock down-regulates HSP production, which is necessary for thermotolerance [81].

In pulmonary artery smooth muscle cells, Rathore et al. [77] suggest that hypoxia-driven mitochondrial ROS signaling triggers NADPH oxidase activity. This would imply a mechanism by which both mitochondria and cytosol contribute to the increase in ROS production and signaling during low $\mathrm{O}_{2}$, at least in animals.

\section{ROS is a common theme in low oxygen and heat stress}

\subsection{ROS-mediated activation of heat shock response (HSR)}

Heat is one of the most serious abiotic stresses for plants due to the extent of irreversible damage it can cause at a morphoanatomical, physiological and biochemical level [113]. Heat stress can strongly interfere and affect normal cell homeostasis by disrupting the metabolic coordination existing within cells and inside a cell, where each pathway is likely to have a temperature optimum in order to work properly [98]. Usually, plants can withstand temperatures of $5-10{ }^{\circ} \mathrm{C}$ above the optimum, specific for each species, by activating the HSR [88] without being stressed [52]. However, when temperatures suddenly rise to high levels, cells can die in a few minutes, as a consequence of a collapse in cellular organization [113].

When plants are subjected to heat stress, they activate a plethora of physiological, molecular and biochemical responses in order to react and survive. General modifications include changes in water status, the accumulation of osmolytes such as proline, hormonal changes, the production of secondary metabolites and alterations in photosynthetic activity, assimilate partitioning, and cell membrane permeability [113]. All these events take a part in a well ordered temporal program to prevent cell death during heat stress (Fig. 2) [113]. At a molecular level, heat stress can lead to genome-wide re-programming events, with de novo synthesis of a heat-related set of proteins, specific to HSR. The synthesis of HSPs represents the typical mechanism of a heat-stress response, which has been observed in various kingdoms, from bacteria to animals [111]. HSPs prevent aggregation, denaturation, misfolding and the degradation of proteins under stress conditions [34]. However, Heat Shock related Genes (HSG) up-regulation has been found to be a general response to biotic [1] and abiotic stress conditions [84,86,99,115], including low $\mathrm{O}_{2}$ stress [7,8,67].

An intimate relationship between HSR and oxidative stress is likely to exist [3], since one of the events occurring in response to high temperatures is ROS production. A burst of $\mathrm{H}_{2} \mathrm{O}_{2}$ has been found to occur in tobacco Bright-Yellow 2 cells undergoing PCD at high temperatures, likely due to an impaired mitochondrial oxidative phosphorylation and imbalance in $\mathrm{H}_{2} \mathrm{O}_{2}$ scavenging [106]. Hydrogen peroxide has been suggested to be required for heat stress and the HSF-dependent expression of genes in Arabidopsis cell cultures, where it likely contributes to the induction of 


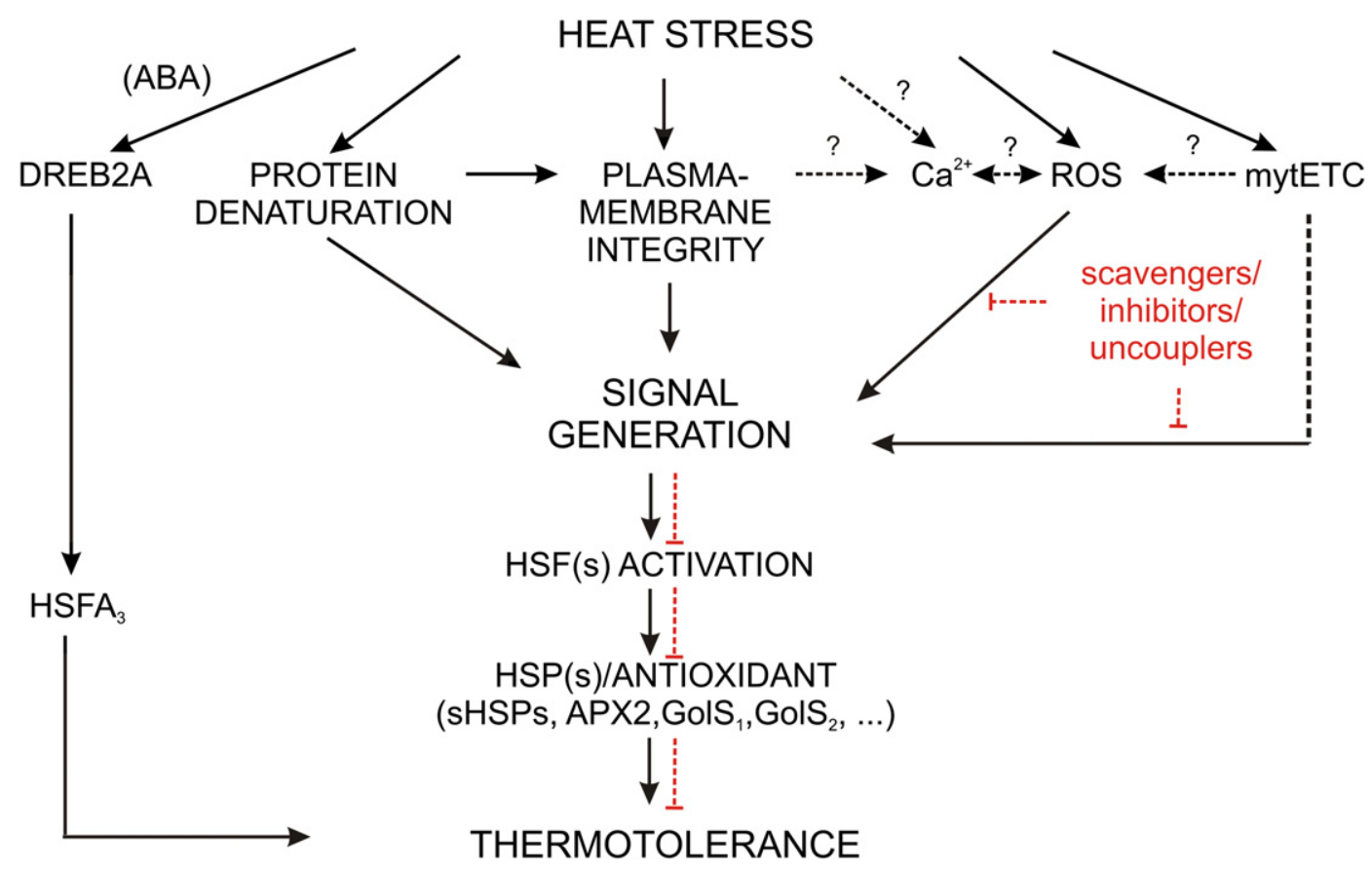

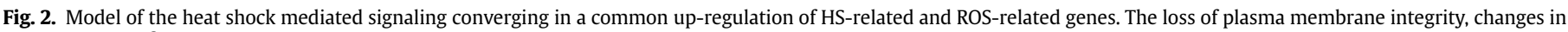

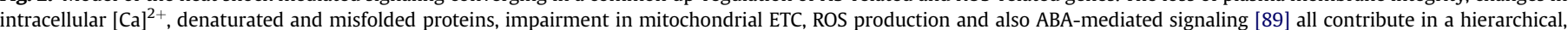

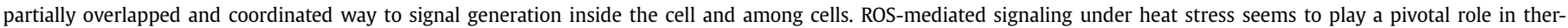

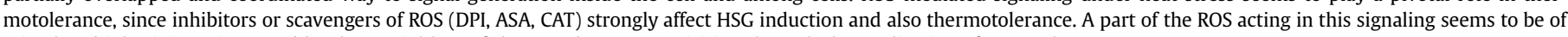
mitochondrial origin as is proved by the partial loss of thermotolerance acquisition through the application of uncouplers.

APX2, HSP17.6 and HSP18.2 [112]. The same phenomenon has been observed in Arabidopsis protoplasts treated with $\mathrm{H}_{2} \mathrm{O}_{2}$ and monitored through a AtHsp18.2-promoter-LUC reporter activity assay [45].

In Arabidopsis cell cultures, experimental evidence has shown that during the early phase of heat stress at both $44{ }^{\circ} \mathrm{C}$ and $37{ }^{\circ} \mathrm{C}$, there is an increase in $\mathrm{H}_{2} \mathrm{O}_{2}$ production, which is necessary for the HSFs-mediated induction of HSGs [112]. This mechanism can be reduced by the application of ROS scavengers/inhibitors, such as ascorbate (ASA) and by DPI [112], thus NADPH oxidase cannot be excluded to have a role in the burst. This idea is also supported by the finding that $r b o h D$ and $r b o h B$ Arabidopsis mutants are defective in thermotolerance [48].

A strong up-regulation of HSPs has been observed in Arabidopsis mutant for ascorbate peroxidise (APX) 1 during light stress [74]. The promoter of this principal $\mathrm{H}_{2} \mathrm{O}_{2}$ scavenging enzyme contains an HSE motif $[21,65,93]$. The APX1-deficient mutant apx1 accumulates more $\mathrm{H}_{2} \mathrm{O}_{2}$ and is significantly sensitive to a heat and drought stress combination $[44,76]$. Experimental evidence has shown that the AtHSF21 transcript is up-regulated in apx1 knockout plants [21,74] and that this $\mathrm{TF}$ is accumulated in the presence of $\mathrm{H}_{2} \mathrm{O}_{2}$ [22]. Another study revealed that transgenic Arabidopsis plants overexpressing HSF3 show increased APX activity during the postheat recovery phase and $A P X 2$ transcript accumulation during increasing temperature $[70,98]$.

All this evidence from biochemical and genetic studies suggests a link between ROS and HSGs and can help to build the hierarchy of genes involved in ROS-mediated stress responses. Miller and Mittler suggested that HSFA4a might rely on a function of TF as a direct ROS sensor, since it likely acts upstream of ZAT12 and APX1 and shows a rapid response to $\mathrm{H}_{2} \mathrm{O}_{2}[21,60]$. However, further studies are necessary to strengthen this hypothesis and to extend it to a more general ROS-related mechanism of sensing, directly mediated by HSFs.

\subsection{Nuclear-mitochondrial cross-talk under heat stress}

Retrograde signaling from organelles communicates a change in cell homeostasis to the nucleus, allowing the nucleus to subsequently modulate anterograde control for plant acclimation [119]. Several possible mechanisms of signaling between the mitochondria and nucleus are thought to occur under stress conditions, involving changes in $\left[\mathrm{Ca}^{2+}\right]$, ROS production, $\mathrm{O}_{2}$ tension and the hyperpolarization of the mitochondrial inner membrane. Although some progress has been made in understanding the communication between the two cellular compartments, this process is still largely unknown $[4,10,75,79,81]$. Recent evidence supports the idea of mitochondrial retrograde regulation as being intimately linked to ROS generation and accumulation [78,79]. During heat stress, $\mathrm{Ca}^{2+}$ uptake by mitochondria and ROS generation have been suggested [79,81]. The ROS source is hypothesized as residing predominantly in the inner membrane of mitochondria where hyperpolarization occurs [81]. Agents that are capable of preventing an increase in inner mitochondrial membrane electrochemical potential also inhibited HSG expression and thermotolerance [81].

In fact, a connection between mitochondrial-mediated signaling and HSG induction has been observed in several plant species. In maize mutants impaired in mitochondrial activity, the expression of some HSGs is up-regulated [46]. Arabidopsis plants transformed with a mitochondrial HSP from Zea mays show the induction of nuclear HSGs [80]. In this heterologous system, the ZmHSP22 transgene was expressed constitutively and it could enter Arabidopsis mitochondria to constitute the mature protein. Under heat stress, Arabidopsis transgenic plants expressing ZmHSP22 display altered expression of several nuclear genes encoding mitochondrial HSPs (AtHSP23.6), and also HSPs localized in chloroplast (AtHSP25.3 and AtHSP70.6) and cytosol (AtHSP17.4 and AtHSP70.1). In addition, Arabidopsis ZmHSP22 plants showed improved thermotolerance [80]. These results suggest that a heat-induced mitochondrial 
retrograde signal via ROS production could regulate HSGs expression, and thus also tolerance.

\subsection{Is heat-anoxia cross tolerance a ROS-mediated mechanism?}

Plant responses to different (a)biotic stress conditions include a transcriptional modulation that is regulated by the presence of cis motifs on the promoter region of stress-responsive genes. Of these motifs, HSEs are represented in several stress-related genes [21,60,65,82]. Indeed, Arabidopsis seedlings show the expression of HSFs and HSP family members under different stress conditions, such as heat, cold, osmotic stress, salt, drought, genotoxicity, ultraviolet light, oxidative stress, wounding, pathogen infection [99], and also $\mathrm{O}_{2}$ deprivation [7,8,67].

This molecular communion might support cross-tolerance, which allows plants to adapt/acclimate to a range of different stresses after exposure to a specific stress [71]. As sessile organisms, plants are usually needed to counteract a combination of (a)biotic stress conditions in nature [36]. In this cross-mechanism, ROS are believed to play a predominant role [71].

Evidence suggesting the central role of ROS in general stress responses is the increase in tolerance of plants treated with $\mathrm{H}_{2} \mathrm{O}_{2}$. Rice seedlings exposed to low levels of $\mathrm{H}_{2} \mathrm{O}_{2}$ are more tolerant to heat and salt stress [105]. Tobacco plants sprayed with $\mathrm{H}_{2} \mathrm{O}_{2}$ increase their tolerance against oxidative stress generated by high light intensities or the catalase inhibitor aminotriazole (AT) [28]. In addition, Arabidopsis plantlets pre-treated with $\mathrm{H}_{2} \mathrm{O}_{2}$ are more tolerant to anoxia [8]. Indeed, HSG induction has a conserved response to $\mathrm{H}_{2} \mathrm{O}_{2}$ in various kingdoms [107]. During both anoxia and heat stress, an increase in $\mathrm{H}_{2} \mathrm{O}_{2}$ production has been observed [8]. Anoxia-driven ROS-mediated signaling probably overlaps with heat-mediated signaling, with HSGs commonly regulated in a ROSdependent way.

Several HSGs are commonly up-regulated when Arabidopsis seedlings are exposed to anoxia, heat, and heat followed by anoxia [8]. Of these, some transcripts (e.g. ZAT12 and AOX1a) belong to the oxidative-responsive gene group [26,42,107]. This might explain the cross-tolerance between heat and anoxia and the higher tolerance of HSFA2 over-expressing plants to anoxia [7,8]. Mild heat pre-treatment, in addition to hypoxic treatment, increases tolerance to anoxia in Arabidopsis [7]. Moreover, hsfA2 mutants do not cross-acclimate to anoxia following mild heat pre-treatment [8].

This heat-mediated cross tolerance event in Arabidopsis is likely to be independent of a low $\mathrm{O}_{2}$ metabolism and the shift towards the adaptive fermentative pathway, since anoxic acclimation through a mild heat stress has also been found to be present in adh mutants impaired in the alcoholic fermentation pathway [7,24]. In fact, OXHSFA2 show a constitutive higher expression of target HSGs (e.g. HSP25.3-P, HSP18.2-Cl, APX2) but not of anaerobic genes (e.g. ADH1 and SUS4) [8]. In animal systems, a moderate heat shock is involved in increased resistance to hypoxia, suggesting the involvement of HSPs in cross-tolerance heat versus low $\mathrm{O}_{2}$ [92]. In addition, the constitutive expression of some HSPs in turtle hearts has been associated with an increase in anoxia tolerance [16].

\section{Concluding remarks}

Although the role of ROS as secondary messengers in plant responses to $\mathrm{O}_{2}$ deprivation is controversial, a common modulation of transcripts related to the redox-metabolism has been observed. A striking overlap between the molecular signaling produced under low $\mathrm{O}_{2}$ and heat stress has also been found, concomitantly with cross tolerance when anoxia takes place after mild heat stress treatment.
Both anoxia and heat have been shown to induce the transcription of HSGs. The heat shock TF HSFA2 likely plays a central role in activating the HSP pathway, which then results in enhanced plant tolerance to both $\mathrm{O}_{2}$ deprivation and heat. Results from mammalian and yeast cells suggest that $\mathrm{HSF}$ are sensors of $\mathrm{H}_{2} \mathrm{O}_{2}$, a model that still awaits confirmation for plants. Indeed, an oxidative burst has been observed after both $\mathrm{O}_{2}$ deprivation and heat, thus supporting the link between $\mathrm{H}_{2} \mathrm{O}_{2}$ production and HSFs activation.

However, many questions remain. The production site of ROS under these stresses is still being debated, although evidence from both the plant and animal kingdoms supports both plasma membrane NADPH oxidase and mitochondria ETC as principal sources. In the latter case, a fascinating area of research regards the idea of mitochondrial retrograde signaling in the control of plant acclimation to stress. The mechanisms that finally lead to heat-anoxia cross-tolerance and acclimation remain elusive and further research is needed to elucidate this link.

\section{Financial source}

This work was supported by Scuola Superiore Sant'Anna.

\section{References}

[1] P. Agudelo-Romero, P. Carbonell, F. de la Iglesia, J. Carrera, G. Rodrigo, A. Jaramillo, M.A. Pérez-Amador, S.F. Elena, Changes in the gene expression profile of Arabidopsis thaliana after infection with Tobacco etch virus, Virol. J. 5 (2008) 92.

[2] R. Ahlfors, V. Macioszek, J. Rudd, M. Brosché, R. Schlichting, D. Scheel, J. Kangasjärvi, Stress hormone-independent activation and nuclear translocation of mitogen-activated protein kinases in Arabidopsis thaliana during ozone exposure, Plant J. 40 (2004) 512-522.

[3] S.-G. Ahn, D.J. Thiele, Redox regulation of mammalian heat shock factor 1 is essential for Hsp gene activation and protection from stress, Genes Dev. 17 (2003) 516-528.

[4] J. Bailey-Serres, R. Chang, Sensing and signalling in response to oxygen deprivation in plants and other organisms, Ann. Bot. 96 (2005) 507-518.

[5] J. Bailey-Serres, L.A.C.J. Voesenek, Flooding stress: acclimations and genetic diversity, Annu. Rev. Plant Biol. 59 (2008) 313-339.

[6] S.K. Baniwal, K. Chan, K.-D. Scharf, L. Nover, Role of heat stress transcription factor HsfA5 as specific repressor of HsfA4, J. Biol. Chem. 282 (2007) 3605-3613.

[7] V. Banti, E. Loreti, G. Novi, A. Santaniello, A. Alpi, P. Perata, Heat acclimation and cross-tolerance against anoxia in Arabidopsis, Plant Cell Environ. 31 (2008) 1029-1037.

[8] V. Banti, F. Mafessoni, E. Loreti, A. Alpi, P. Perata, The heat-inducible transcription factor HsfA2 enhances anoxia tolerance in Arabidopsis, Plant Physiol. 152 (2010) 1471-1483.

[9] A. Baxter-Burrell, Z. Yang, P.S. Springer, J. Bailey-Serres, RopGAP4-dependent Rop GTPase rheostat control of Arabidopsis oxygen deprivation tolerance, Science 296 (2002) 2026-2028.

[10] G. Biswas, M. Guha, N.G. Avadhani, Mitochondria-to-nucleus stress signalling in mammalian cells: nature of nuclear gene targets, transcription regulation, and induced resistance to apoptosis, Gene 18 (2005) 132-139.

[11] O. Blokhina, K.V. Fagerstedt, Oxidative metabolism, ROS and NO under oxygen deprivation, Plant Physiol. Biochem. 48 (2010) 359-373.

[12] C. Branco-Price, K.A. Kaiser, C.J. Jang, C.K. Larive, J. Bailey-Serres, Selective mRNA translation coordinates energetic and metabolic adjustments to cellular oxygen deprivation and reoxygenation in Arabidopsis thaliana, Plant J. 56 (2008) 743-755.

[13] C. Branco-Price, R. Kawaguchi, R.B. Ferreira, J. Bailey-Serres, Genome-wide analysis of transcript abundance and translation in Arabidopsis seedlings subjected to oxygen deprivation, Ann. Bot. 96 (2005) 647-660.

[14] N.S. Chandel, E. Maltepe, E. Goldwasser, C.E. Mathieu, M.C. Simon P.T. Schumacker, Mitochondrial reactive oxygen species trigger hypoxiainduced transcription, Proc. Natl. Acad. Sci. U.S.A. 95 (1998) 11715-11720.

[15] N.S. Chandel, D.S. McClintock, C.E. Feliciano, T.M. Wood, J.A. Melendez, A.M. Rodriguez, P.T. Schumacker, Reactive oxygen species generated at mitochondrial complex III stabilize hypoxia-inducible factor-1alpha during hypoxia: a mechanism of $\mathrm{O}_{2}$ sensing, J. Biol. Chem. 275 (2000) 25130-25138.

[16] J. Chang, A.A. Knowlton, J.S. Wasser, Expression of heat shock proteins in turtle and mammal hearts: relationship to anoxia tolerance, Am. J. Physiol. Regul. Integr. Comp. Physiol. 278 (2000) 209-214.

[17] R. Chang, C.J.H. Jang, C. Branco-Price, P. Nghiem, J. Bailey-Serres, Transient MPK6 activation in response to oxygen deprivation and reoxygenation is mediated by mitochondria and aids seedling survival in Arabidopsis, Plant Mol. Biol. 78 (2012) 109-122. 
[18] K.Y. Chan-Schaminet, S.K. Baniwal, D. Bublak, L. Nover, K.-D. Scharf, Specific interaction between tomato HsfA1 and HsfA2 creates hetero-oligomeric superactivator complexes for synergistic activation of heat stress gene expression, J. Biol. Chem. 284 (2009) 20848-20857.

[19] T.D. Colmer, Long-distance transport of gases in plants: a perspective on internal aeration and radial oxygen loss from roots, Plant Cell Environ. 26 (2003) 17-37.

[20] M.C.H. Cox, F.F. Millenaar, Y.E.M.D.J. Van Berkel, A.J.M. Peeters, L.A.C.J. Voesenek, Plant movement. Submergence-induced petiole elongation in Rumex palustris depends on hyponastic growth, Plant Physiol. 132 (2003) $282-291$.

[21] S. Davletova, L. Rizhsky, H. Liang, Z. Shengqiang, D.J. Oliver, J. Coutu, V. Shulaev, K. Schlauch, R. Mittler, Cytosolic ascorbate peroxidise 1 is a central component of the reactive oxygen gene network of Arabidopsis, Plant Cell 17 (2005) 268-281.

[22] S. Davletova, K. Schlauch, J. Coutu, R. Mittler, The zinc-finger protein Zat12 plays a central role in reactive oxygen and abiotic stress signaling in Arabidopsis, Plant Physiol. 133 (2005) 847-856.

[23] L. Denness, J.F. McKenna, C. Segonzac, A. Wormit, P. Madhou, M. Bennett, J. Mansfield, C. Zipfel, T. Hamann, Cell wall damage induced lignin biosynthesis is regulated by a ROS- and jasmonic acid dependent process in Arabidopsis thaliana, Plant Physiol. 156 (2011) 1364-1374.

[24] M.H. Ellis, E.S. Dennis, W.J. Peacock, Arabidopsis roots and shoots have different mechanisms for hypoxic stress tolerance, Plant Physiol. 119 (1999) 57-64.

[25] T. Fukao, K. Xu, P.C. Ronald, J. Bailey-Serres, A variable cluster of ethylene response factor-like genes regulates metabolic and developmental acclimation responses to submergence in rice, Plant Cell 18 (2006) 2021-2034.

[26] I. Gadjev, S. Vanderauwera, T.S. Gechev, C. Laloi, I.N. Minkov, V. Shulaev, K. Apel, D. Inzé, R. Mittler, F. Van Breusegem, Transcriptomic footprints disclose specificity of reactive oxygen species signaling in Arabidopsis, Plant Physiol. 141 (2006) 436-445.

[27] M. Garzón, K. Eifler, A. Faust, H. Scheel, K. Hofmann, C. Koncz, A. Yephremov, A. Bachmair, PRT6/At5g02310 encodes an Arabidopsis ubiquitin ligase of the $\mathrm{N}$-end rule pathway with arginine specificity and is not the CER3 locus, FEBS Lett. 581 (2007) 3189-3196.

[28] T. Gechev, I. Gadjev, F. Van Breusegem, D. Inzè, S. Dukiandjiev, V. Toneva, I. Minkov, Hydrogen peroxide protects tobacco from oxidative stress by inducing a set of antioxidant enzymes, Cell Mol. Life Sci. 59 (2002) 708-714.

[29] P. Geigenberger, Response of plant metabolism to too little oxygen, Curr. Opin. Plant Biol. 6 (2003) 247-256.

[30] D.J. Gibbs, S.C. Lee, N.M. Isa, S. Gramuglia, T. Fukao, G.W. Bassel, C.S. Correia, F. Corbineau, F.L. Theodoulou, J. Bailey-Serres, M.J. Holdsworth, Homeostatic response to hypoxia is regulated by the $\mathrm{N}$-end rule pathway in plants, Nature 479 (2011) 415-418.

[31] E. Graciet, F. Walter, D. Ó Maoiléidigh, S. Pollmann, E.M. Meyerowitz, A. Varshavsky, F. Wellmer, The N-end rule pathway controls multiple functions during Arabidopsis shoot and leaf development, Proc. Natl. Acad. Sci. U.S.A. 106 (2009) 13618-13623.

[32] R.B. Hamanaka, N.S. Chandel, Mitochondrial reactive oxygen species regulate hypoxic signaling, Curr. Opin. Cell Biol. 21 (2009) 894-899.

[33] D. Han, F. Antunes, R. Canali, D. Rettori, E. Cadenas, Voltage-dependent anion channels control the release of the superoxide anion from mitochondria to cytosol, J. Biol. Chem. 278 (2003) 5557-5563.

[34] F.U. Hartl, Molecular chaperones in cellular protein folding, Nature 381 (1996) 571-579.

[35] Y. Hattori, K. Nagai, S. Furukawa, X.J. Song, R. Kawano, H. Sakakibara, J. Wu, T. Matsumoto, A. Yoshimura, H. Kitano, M. Matsuoka, H. Mori, M. Ashikari, The ethylene response factors SNORKEL1 and SNORKEL2 allow rice to adapt to deep water, Nature 460 (2009) 1026-1030.

[36] T. Hirayama, K. Shinozaki, Research on plant abiotic stress response in the post-genome era: past, present and future, Plant J. 61 (2010) 1041-1052.

[37] L.E. Huang, J. Gu, M. Schau, H.F. Bunn, Regulation of hypoxia-inducible factor 1alpha is mediated by an $\mathrm{O}_{2}$-dependent degradation domain via the ubiquitin-proteasome pathway, Proc. Natl. Acad. Sci. U.S.A. 95 (1998) 7987-7992.

[38] M. Ivan, K. Kondo, H. Yang, W. Kim, J. Valiando, M. Ohh, A. Salic, J.M. Asara, W.S. Lane, W.G.J. Kaelin, HIF-alpha targeted for VHL-mediated destruction by proline hydroxylation: implications for $\mathrm{O}_{2}$ sensing, Science 292 (2001) 464-468.

[39] P. Jaakkola, D.R. Mole, Y.M. Tian, M.I. Wilson, J. Gielbert, S.J. Gaskell, A.v. Kriegsheim, H.F. Hebestreit, M. Mukherji, C.J. Schofield, P.H. Maxwell C.W. Pugh, P.J. Ratcliffe, Targeting of HIF-alpha to the von Hippel-Lindau ubiquitylation complex by $\mathrm{O}_{2}$-regulated prolyl hydroxylation, Science 292 (2001) 468-472.

[40] C. Jonak, H. Nakagami, H. Hirt, Heavy metal stress. Activation of distinct mitogen-activated protein kinase pathways by copper and cadmium, Plant Physiol. 136 (2004) 3276-3283.

[41] K.-H. Jung, Y.-S. Seo, H. Walia, P. Cao, T. Fukao, P.E. Canlas, F. Amonpant Bailey- J. Serres, P.C. Ronald, The submergence tolerance regulator Sub1A mediates stress-responsive expression of AP2/ERF transcription factors, Plant Physiol. 152 (2010) 1674-1692.

[42] H.S. Kim, E.C. Snesrud, L.P. Moy, B.J. Haas, W.C. Nierman, J. Quackenbush, Transcriptional divergence of the duplicated oxidative stress-responsive genes in the Arabidopsis genome, Plant J. 41 (2005) 212-220.
[43] E.J. Klok, I.W. Wilson, D. Wilson, S.C. Chapman, R.M. Ewing, S.C. Somerville, W.J. Peacock, R. Dolferus, E.S. Dennis, Expression profile analysis of the lowoxygen response in Arabidopsis root cultures, Plant Cell 14 (2002) 2481-2494.

[44] S. Koussevitzky, N. Suzuki, S. Huntington, L. Armijo, W. Sha, D. Cortes, V. Shulaev, R. Mittler, Ascorbate Peroxidase 1 plays a key role in the response of Arabidopsis thaliana to stress combination, J. Biol. Chem. 283 (2008) 34197-34203.

[45] Y. Kovtun, W.L. Chiu, G. Tena, J. Sheen, Functional analysis of oxidative stress-activated mitogen-acitvated protein kinase cascade in plants, Proc. Natl. Acad. Sci. U.S.A. 97 (2000) 2940-2945.

[46] E.V. Kuzmin, O.V. Karpova, T.E. Elthon, K.J. Mewton, Mitochondrial respiratory deficiencies signal up-regulation of genes for heat shock proteins, J. Biol. Chem. 279 (2004) 20672-20677.

[47] J.M. Kwak, I.C. Mori, Z.M. Pei, N. Leonhardt, M.A. Torres, J.L. Dangl, R.E. Bloom, S. Bodde, J.D. Jones, J.I. Schroeder, NADPH oxidase AtrbohD and AtrbohF genes function in ROS-dependent ABA signaling in Arabidopsis, EMBO J. 22 (2003) 2623-2633.

[48] J. Larkindale, J.D. Hall, M.R. Knight, E. Vierling, Heat stress phenotypes of Arabidopsis mutants implicate multiple signaling pathways in the acquisition of thermotolerance, Plant Physiol. 138 (2005) 882-897.

[49] R. Lasanthi-Kudahettige, L. Magneschi, E. Loreti, S. Gonzali, F. Licausi, G. Novi, O. Beretta, F. Vitulli, A. Alpi, P. Perata, Transcript profiling of the anoxic rice coleoptile, Plant Physiol. 144 (2007) 218-231.

[50] S. Lee, T. Carlson, N. Christian, K. Lea, J. Kedzie, J.P. Reilly, J.J. Bonner, The yeast heat shock transcription factor changes conformation in response to superoxide and temperature, Mol. Biol. Cell 11 (2000) 1753-1764.

[51] S.C. Lee, A. Mustroph, R. Sasidharan, D. Vashisht, O. Pedersen, T. Oosumi, L.A.C.J. Voesenek, J. Bailey-Serres, Molecular characterization of the submergence response of the Arabidopsis thaliana ecotype Columbia, New Phytol. 190 (2011) 457-471.

[52] A. Leone, C. Perrotta, B. Maresca, Tolerance to heat stress: current strategies and new emergent insights, in: L. Sanità di Toppi, B. Pawlik-Skowronska (Eds.), Abiotic Stress in Plants, Kluwer Academic Publisher, 2003, pp. 1-22.

[53] F. Licausi, Regulation of the molecular response to oxygen limitations in plants, New Phytol. 190 (2011) 550-555.

[54] F. Licausi, M. Kosmacz, D.A. Weits, B. Giuntoli, F.M. Giorgi, L.A. Voesenek, P. Perata, J.T. van Dongen, Oxygen sensing in plants is mediated by an N-end rule pathway for protein destabilization, Nature 479 (2011) 419-422.

[55] F. Licausi, P. Perata, Low oxygen signaling and tolerance in plants, Adv. Bot. Res. 50 (2009) 189-198.

[56] F. Licausi, J.T. van Dongen, B. Giuntoli, G. Novi, A. Santaniello, P. Geigenberger, P. Perata, HRE1 and HRE2, two hypoxia-inducible ethylene response factors, affect anaerobic responses in Arabidopsis thaliana, Plant J. 62 (2011) 302-315.

[57] F. Licausi, D.A. Weits, B.D. Pant, W.R. Scheible, P. Geigenberger, J.T. van Dongen, Hypoxia responsive gene expression is mediated by various subsets of transcription factors and miRNAs that are determined by the actual oxygen availability, New Phytol. 190 (2011) 442-456.

[58] H.-c. Liu, H.-t. Liao, Y.-y. Charng, The role of class A1 heat shock factors (HSFA1s) in response to heat and other stresses in Arabidopsis, Plant Cell Environ. 34 (2011) 738-751.

[59] E. Loreti, A. Poggi, G. Novi, A. Alpi, P. Perata, A genome-wide analysis of the effects of sucrose on gene expression in Arabidopsis seedlings under anoxia, Plant Physiol. 137 (2005) 1130-1138.

[60] G. Miller, R. Mittler, Could heat shock transcription factors function as hydrogen peroxide sensors in plants? Ann. Bot. 98 (2006) 279-288.

[61] G. Miller, V. Shulaev, R. Mittler, Reactive oxygen signaling and abiotic stress, Physiol. Plantarum 133 (2008) 481-489.

[62] G. Miller, K. Schlauch, R. Tam, D. Cortes, M.A. Torres, V. Shulaev, J.L. Dangl, R. Mittler, The plant NADPH oxidase RBOHD mediates rapid systemic signaling in response to diverse stimuli, Sci. Signal. 2 (2009) ra45.

[63] R. Mittler, S. Vanderauwera, M. Gollery, F. Van Breusegem, Reactive oxygen gene network of plants, Trends Plant Sci. 9 (2004) 490-498.

[64] R. Mittler, S. Vanderauwera, N. Suzuki, G. Miller, V.B. Tognetti, K. Vandepoele, M. Gollery, V. Shulaev, F. Van Breusegem, ROS signaling: the new wave? Trends Plant Sci. 16 (2011) 300-309.

[65] R. Mittler, B.A. Zilinskas, Molecular cloning and characterization of a gene encoding pea cytosolic ascorbate peroxidise, J. Biol. Chem. 267 (1992) 21802-21807.

[66] F.L. Muller, Y. Liu, H. Van Remmen, Complex III releases superoxide to both sides of the inner mitochondrial membrane, J. Biol. Chem. 279 (2004) 49064-49073.

[67] A. Mustroph, S.C. Lee, T. Oosumi, M.E. Zanetti, H. Yang, K. Ma, A. YaghoubiMasihi, T. Fukao, J. Bailey-Serres, Cross-kingdom comparison of transcriptomic adjustments to low-oxygen stress highlights conserved and plant-specific responses, Plant Physiol. 152 (2010) 1484-1500.

[68] Y. Ogasawara, H. Kaya, G. Hiraoka, F. Yumoto, S. Kimura, Y. Kadota, H. Hishinuma, E. Senzaki, S. Yamagoe, K. Nagata, M. Nara, K. Suzuki, M. Tanokura, K. Kuchitsu, Synergistic activation of the Arabidopsis NADPH oxidase AtrbohD by $\mathrm{Ca}^{2+}$ and phosphorylation, J. Biol. Chem. 283 (2008) 8885-8892.

[69] M. Ohh, C.W. Park, M. Ivan, M.A. Hoffman, T.Y. Kim, L.E. Huang, N. Pavletich, V. Chau, W.G. Kaelin, Ubiquitination of hypoxia-inducible factor requires 
direct binding to the beta-domain of the von Hippel-Lindau protein, Nat. Cell Biol. 2 (2000) 423-427.

[70] I.I. Panchuk, R.A. Volkov, F. Schöffl, Heat stress- and heat shock transcription factor-dependent expression and activity of ascorbate peroxidise in Arabidopsis, Plant Physiol. 129 (2002) 838-853.

[71] G.M. Pastori, C.H. Foyer, Common components, networks, and pathways of cross-tolerance to stress. The central role of "Redox" and abscisic acidmediated controls, Plant Physiol. 129 (2002) 460-468.

[72] S. Parlanti, N.P. Kudahettige, L. Lombardi, A. Mensuali-Sodi, A. Alpi, P. Perata, C. Pucciariello, Distinct mechanisms for aerenchyma formation in leaf sheaths of rice genotypes displaying a quiescence or escape strategy for flooding tolerance, Ann. Bot. 107 (2011) 1335-1343.

[73] P. Perata, A. Alpi, Plant responses to anaerobiosis, Plant Sci. 93 (1993) 1-17.

[74] L. Pnueli, H. Liang, M. Rozenberg, R. Mittler, Growth suppression, altered stomatal responses, and augmented induction of heat shock proteins in cytosolic ascorbate peroxidase (Apx1)-deficient Arabidopsis plants, Plant J. 34 (2003) 187-203.

[75] R.O. Poyton, C.J. Dagsgaard, Mitochondrial-nuclear crosstalk is involved in oxygen-regulated gene expression in yeast, Adv. Exp. Med. Biol. 475 (2000) $177-184$.

[76] D.C. Raitt, A.L. Johnson, A.M. Erkine, K. Makino, B. Morgan, D.S. Gross, L.H. Johnston, The Skn7 response regulator of Saccharomyces cerevisiae interacts with Hsf1 in vivo and is required for the Induction of heat shock genes, Mol. Biol. Cell 11 (2000) 2335-2347.

[77] R. Rathore, Y.M. Zheng, C.F. Niu, Q.H. Liu, A. Korde, Y.S. Ho, Y.X. Wang, Hypoxia activates NADPH oxidase to increase [ROS]i and $\left[\mathrm{Ca}^{2+}\right] \mathrm{i}$ through the mitochondrial ROS-PKCepsilon signaling axis in pulmonary artery smooth muscle cells, Free Radic. Biol. Med. 45 (2008) 1223-1231.

[78] D.M. Rhoads, C.C. Subbaiah, Mitochondrial retrograde regulation in plants, Mitochondrion 7 (2007) 177-194.

[79] D.M. Rhoads, A.L. Umbach, C.C. Subbaiah, J.N. Siedow, Mitochondrial reactive oxygen species. Contribution to oxidative stress and interorganellar signaling, Plant Physiol. 141 (2006) 357-366.

[80] D.M. Rhoads, S.J. White, Y. Zhou, M. Muralidharan, T.E. Elthon, Altered gene expression in plants with constitutive expression of a mitochondrial small heat shock protein suggests the involvement of retrograde regulation in the heat stress response, Physiol. Plant 123 (2005) 435-444.

[81] E.G. Rikhvanov, K.Z. Gamburg, N.N. Varakina, T.M. Rusaleva, I.V. Fedoseeva, E.L. Tauson, I.V. Stupnikova, A.V. Stepanov, G.B. Borovskii, V.K. Voinikov, Nuclear-mitochondrial cross-talk during heat shock in Arabidopsis cell culture, Plant J. 52 (2007) 763-778.

[82] L. Rizhsky, S. Davletova, H. Liang, R. Mittler, The zinc finger protein Zat12 Is required for cytosolic ascorbate peroxidase 1 expression during oxidative stress in Arabidopsis, J. Biol. Chem. 279 (2004) 11736-11743.

[83] M. Rocha, F. Licausi, W.L. Araújo, A. Nunes-Nesi, L. Sodek, A.R. Fernie, J.T. van Dongen, Glycolysis and the tricarboxylic acid cycle are linked by alanine aminotransferase during hypoxia induced by waterlogging of Lotus japonicus, Plant Physiol. 152 (2010) 1501-1513.

[84] K. Saarma, M.T. Tarkka, M. Itävaara, K.V. Fagerstedt, Heat shock protein synthesis is induced by diethyl phthalate but not by di(2-ethylhexyl) phthalate in radish (Raphanus sativus), J. Plant Physiol. 160 (2003) 1001-1010.

[85] M. Sagi, R. Fluhr, Superoxide production by plant homologues of the gp91(phox) NADPH oxidase modulation of activity by calcium and by tobacco mosaic virus infection, Plant Physiol. 126 (2001) 1281-1290.

[86] T.E. Scarpeci, M.I. Zanor, E.M. Valle, Investigating the role of plant heat shock proteins during oxidative stress, Plant Signal. Behav. 3 (2008) 856-857.

[87] K.-D. Scharf, H. Heider, I. Höhfeld, R. Lyck, E. Schmidt, L. Nover, The tomato Hsf system: HsfA2 needs interaction with HsfA1 for efficient nuclear import and may be localized in cytoplasmic heat stress granules, Mol. Cell. Biol. 18 (1998) 2240-2251.

[88] F. Schöffl, R. Prandl, A. Reindt, Molecular response to heat stress, in: K. Shinozaki, K. Yamaguchi-Shinozaki (Eds.), Molecular Responses to Cold, Drought, Heat and Salt Stress in Higher Plants, R.G. Landes Co., Austin, Texas, 1999, pp. 81-98.

[89] F. Schramm, J. Larkindale, E. Kiehlmann, A. Ganguli, G. Englich, E. Vierling, P. von Koskull-Döring, A cascade of transcription factor DREB2A and heat stress transcription factor HsfA3 regulates the heat stress response of Arabidopsis, Plant J. 53 (2008) 264-274.

[90] J.C. Sedbrook, P.J. Kronebusch, G.G. Borisy, A.J. Trewavas, P.H. Masson, Transgenic AEQUORIN reveals organ-specific cytosolic $\mathrm{Ca}^{2+}$ responses to anoxia and Arabidopsis thaliana seedlings, Plant Physiol. 111 (1996) $243-257$

[91] G.L. Semenza, Hypoxia-inducible factor 1 (HIF-1) pathway, Sci. STKE 2007 (2007) $\mathrm{cm} 8$.

[92] L.H.E.H. Snoeckx, R.N. Cornelussen, F.A. Van Nieuwenhoven, R.S. Reneman, G.J. Van der Vusse, Heat shock proteins and cardiovascular pathophysiology, Physiol. Rev. 81 (2001) 1461-1497.

[93] S. Storozhenko, P.D. Pauw, M.V. Montagu, D. Inzè, S. Kushnir, The heat-shock element is a functional component of the Arabidopsis APX1 gene promoter, Plant Physiol. 118 (1998) 1005-1014.
[94] C.C. Subbaiah, D.S. Bush, M.M. Sachs, Elevation of cytosolic calcium precedes anoxic gene expression in maize suspension-cultured cells, Plant Cell 6 (1994) 1747-1762.

[95] C.C. Subbaiah, J. Zhang, M.M. Sachs, Involvement of intracellular calcium in anaerobic gene expression and survival of maize seedlings, Plant Physiol. 105 (1994) 369-376.

[96] N. Suzuki, S. Koussevitzky, R. Mittler, G. Miller, ROS and redox signalling in the response of plants to abiotic stress, Plant Cell Environ. 35 (2012) 259-270.

[97] N. Suzuki, G. Miller, J. Morales, V. Shulaev, M.A. Torres, R. Mittler, Respiratory burst oxidases: the engines of ROS signaling, Curr. Opin. Plant Biol. 14(2011)1-9.

[98] N. Suzuki, R. Mittler, Reactive oxygen species and temperature stresses: a delicate balance between signaling and destruction, Physiol. Plant 126 (2006) 45-51.

[99] W. Swindell, M. Huebner, A.P. Weber, Transcriptional profiling of Arabidopsis heat shock proteins and transcription factors reveals extensive overlap between heat and non-heat stress response pathway, BMC Genomics 8 (2007) 125.

[100] S. Takeda, C. Gapper, H. Kaya, E. Bell, K. Kuchitsu, L. Dolan, Local positive feedback regulation determines cell shape in root hair cells, Science 319 (2008) 1241-1244.

[101] M.A. Torres, J.L. Dangl, J.D. Jones, Arabidopsis gp91 phox homologues AtrbohD and AtrbohF are required for accumulation of reactive oxygen intermediates in the plant defense response, Proc. Natl. Acad. Sci. U.S.A. 99 (2002) 517-522.

[102] M.A. Torres, J.L. Dangl, J.D.G. Jones, Pathogen-induced, NADPH oxidasederived reactive oxygen intermediates suppress spread of cell death in Arabidopsis thaliana, Nat. Genet. 37 (2005) 1130-1134.

[103] M.A. Torres, J.L. Dangl, Functions of the respiratory burst oxidase in biotic interactions, abiotic stress and development, Curr. Opin. Plant Biol. 8 (2005) 397-403.

[104] J.F. Turrens, Mitochondrial formation of reactive oxygen species, J. Physiol. 552 (2003) 335-344.

[105] A. Uchida, A.T. Jagendorf, T. Hibino, T. Takabe, T. Takabe, Effects of hydrogen peroxide and nitric oxide on both salt and heat stress tolerance in rice, Plant Sci. 163 (2002) 515-523.

[106] R.A. Vacca, M.C. de Pinto, D. Valenti, S. Passarella, E. Marra, L. De Gara, Production of reactive oxygen species, alteration of cytosolic ascorbate peroxidase, and impairment of mitochondrial metabolism are early events in heat shock induced programmed cell death in tobacco Bright-Yellow 2 cell, Plant Physiol. 134 (2004) 1100-1112.

[107] K. Vandenbroucke, S. Robbens, K. Vandepoele, D. Inzè, Y. Van de Peer, F. Van Breusegem, Hydrogen peroxide-induced gene expression across kingdoms: a comparative analysis, Mol. Biol. Evol. 25 (2008) 507-516.

[108] J.T. van Dongen, A. Fröhlich, S.J. Ramírez-Aguilar, N. Schauer, A.R. Fernie A. Erban, J. Kopka, J. Clark, A. Langer, P. Geigenberger, Transcript and metabolite profiling of the adaptive response to mild decreases in oxygen concentration in the roots of Arabidopsis plants, Ann. Bot. 103 (2008) 269-280.

[109] B.B. Vartapetian, M. Jackson, Plant adaptations to anaerobic stress, Ann. Bot. 79 (1997) 3-20.

[110] M.L. Vidoz, E. Loreti, A. Mensuali, A. Alpi, P. Perata, Hormonal interplay during adventitious root formation in flooded tomato plants, Plant J. 63 (2010) 551-562.

[111] E. Vierling, The role of heat shock proteins in plants, Annu. Rev. Plan. Phys. 42 (1991) 579-620.

[112] R.A. Volkov, I.I. Panchuck, P.M. Mullineaux, F. Schöffl, Heat stress-induced $\mathrm{H}_{2} \mathrm{O}_{2}$ is required for effective expression of heat shock genes in Arabidopsis, Plant Mol. Biol. 61 (2006) 733-746.

[113] A. Wahid, S. Gelani, M. Ashraf, M.R. Foolad, Heat tolerance in plants: an overview, Environ. Exp. Bot. 61 (2007) 199-223.

[114] P. Wang, Y. Du, Y. Li, D. Ren, C.P. Song, Hydrogen peroxide-mediated activation of MAP kinase 6 modulates nitric oxide biosynthesis and signal transduction in Arabidopsis, Plant Cell 22 (2010) 2981-2998.

[115] W. Wang, B. Vinocur, O. Shoseyov, A. Altman, Role of plant heat-shock proteins and molecular chaperones in the abiotic stress response, Trends Plant Sci. 9 (2004) 244-252.

[116] J. Ward, Oxygen sensors in context, Biochim. Biophys. Acta 1777 (2008) 1-14

[117] G.B. Waypa, J.D. Marks, R. Guzy, P.T. Mungai, J. Schriewer, D. Dokic P.T. Schumacker, Hypoxia triggers subcellular compartmental redox signaling in vascular smooth muscle cells, Circ. Res. 106 (2010) 526-535.

[118] G.B. Waypa, P.T. Schumacker, Hypoxia-induced changes in pulmonary and systemic vascular resistance: where is the $\mathrm{O}_{2}$ sensor? Respir. Physiol. Neurobiol. 174 (2010) 201-211.

[119] J.D. Woodson, J. Chory, Coordination of gene expression between organellar and nuclear genomes, Nat. Rev. Genet. 9 (2008) 383-395.

[120] S. Yoshida, M. Ito, J. Callis, I. Nishida, A. Watanabe, A delayed leaf senescence mutant is defective in arginyl-tRNA:protein arginyltransferase, a component of the N-end rule pathway in Arabidopsis, Plant J. 32 (2002) 129-137.

[121] T. Yuasa, K. Ichimura, T. Mizoguchi, K. Shinozaki, Oxidative stress activates ATMPK6, an Arabidopsis homologue of MAP kinase, Plant Cell Physiol. 42 (2001) 1012-1016.

[122] M. Zhong, A. Orosz, C. Wu, Direct sensing of heat and oxidation by Drosophila heat shock transcription factor, Mol. Cell (1998) 101-108. 